\section{CTLA-4-Hemmer bei SCLC-Patienten}

\begin{abstract}
Beim kleinzelligen Lungenkarzinom im ausgedehnten Stadium (extensive stage, SCLC-ES) sind die Behandlungserfolge beschränkt. Ob Ipilimumab die Ergebnisse verbessern kann, wurde in einer Phase-III-Studie untersucht.
\end{abstract}

$P$ a atienten mit SCLC-ES haben geringe Überlebenschancen, selbst wenn sie in der Erstlinie eine Chemotherapie mit Etoposid und einem Platinderivat erhalten. Die Suche nach effektiveren Therapien war in den vergangenen Jahren nicht erfolgreich, doch deuten die hohe Zahl somatischer Mutationen beim SCLC und die Präsenz paraneoplastischer Autoimmun-Syndrome auf eine Immunogenität des Tumors hin. Immuncheckpointinhibitoren zeigten bereits Wirkung. In der vorliegenden multizentrischen Phase-III-Studie wurde daher bei Patienten mit frisch diagnostiziertem SCLC-ES der CTLA-4 („cytotoxic $\mathrm{T}$ lymphocyte-associated antigen 4")-Inhibitor Ipilimumab erprobt.

In der verblindeten Studie erhielten alle Patienten eine Chemotherapie aus
Etoposid und einem Platinderivat sowie zusätzlich entweder $10 \mathrm{mg} / \mathrm{kg}$ Ipilimumab oder Placebo. Dabei wurde die Chemotherapie in den Zyklen 1-4 gegeben; die Zusatzbehandlung mit Ipilimumab oder Placebo begann erst in Zyklus 3 und dauerte längstens bis Zyklus 6. Es schloss sich aber noch eine Erhaltungstherapie mit Ipilimumab oder Placebo für 12 Wochen an. Primärer Endpunkt war das Gesamtüberleben der insgesamt 954 Patienten, die mindestens eine Dosis der Studienmedikation erhalten hatten.

Das mediane Gesamtüberleben betrug 11,0 Monate im Ipilimumab- gegenüber 10,9 Monaten im Placeboarm (Hazard Ratio [HR] 0,94; $\mathrm{p}=0,3775)$, das mediane progressionsfrie Überleben 4,6 gegenüber 4,4 Monaten (HR 0,85).
Häufigkeit und Schwere behandlungsbedingter Toxizitäten waren vergleichbar, lediglich Diarrhö, Hautausschlag und Kolitis unter Ipilimumab häufiger. Auch unterbrachen mehr Patienten aus diesem Arm die Behandlung (18 vs. $2 \%$ ). 5 behandlungsbedingte Todesfälle traten unter Ipilimumab auf, 2 in der Placebogruppe.

Fazit: Die Zugabe von Ipilimumab zur Chemotherapie aus Etoposid und Platin verlängerte das Gesamtüberleben im Vergleich zur alleinigen Chemotherapie bei Patienten mit neu diagnostiziertem SCLC-ES nicht. Allerdings führte Ipilimumab auch nicht zu neuen oder unerwarteten Nebenwirkungen. Womöglich nützt Ipilimumab in Kombination mit anderen Checkpointinhibitoren, etwa den PD-1-Inhibitoren, wie es derzeit getestet wird.

Christina Berndt

Reck M et al. Phase III Randomized Trial of Ipilimumab Plus Etoposide and Platinum Versus Placebo Plus Etoposide and Platinum in Extensive-Stage Small-Cell Lung Cancer. J Clin Oncol. $2016 \mathrm{Jul} 25$. [Epub ahead of print].

\title{
Zweitlinientherapie des SCLC
}

\section{Die Monotherapie mit Topotecan gilt als Behandlung der Wahl beim rezidi- vierten kleinzelligen Lungenkarzinom (SCLC). Dabei gehören SCLC zu den besonders chemosensitiven Tumoren. Wäre eine Kombinationsbehandlung mit verschiedenen Chemotherapeutika daher nicht effektiver?}

P atie tienten mit SCLC werden meist mit Platinregimes behandelt, doch binnen eines Jahres kommt es gemeinhin zu sensitiven oder refraktären Rezidiven. Für das sensitive Rezidiv ist die Topotecan-Monotherapie bisher die einzig zugelassene Behandlung, doch verschiedene Substanzen wie Cisplatin, Etoposid und Irinotecan gelten auch beim rezidivierten SCLC als effektiv. Da der Erfolg von Topotecan in der Zweitlinie zudem überschaubar ist (Ansprechrate 7-24\%, medianes Überleben 25-35 Wochen), wurde in der vorliegenden Studie untersucht, ob eine Kombination die Ergebnisse einer Topotecan-Monotherapie beim sensitiven Rezidiv verbessern kann.

An der Phase-III-Studie nahmen 29 japanische Kliniken teil. 180 Patienten mit SCLC, bei denen mehr als 90 Tage nach erfolgreicher Erstlinienbehandlung ein Rezidiv auftrat, wurden in die Studie aufgenommen und randomisiert. Sie erhielten Topotecan alleine oder kombiniert mit Cisplatin $\left(25 \mathrm{mg} / \mathrm{m}^{2}\right.$ i.v. an Tag 1 und 8), Etoposid $\left(60 \mathrm{mg} / \mathrm{m}^{2}\right.$ i.v. an Tag 1-3), Irinotecan $\left(90 \mathrm{mg} / \mathrm{m}^{2}\right.$ i. v. an Tag 8) und Granulozytenkolonie-stimulierendem Faktor (G-CSF).

Topotecan wurde über 4 Zylen gegeben $\left(1,0 \mathrm{mg} / \mathrm{m}^{2} \mathrm{i}\right.$. v. an den Tagen $1-5$ alle 3 Wochen). Primärer Endpunkt war das Gesamtüberleben.

Nach median 22,7 Monaten Follow-up war das Gesamtüberleben unter der Kombination signifikant länger als unter Topotecan allein (median 18,2 vs. 12,5 Monate; Hazard Ratio 0,67; $\mathrm{p}=0,0079$ ).
Die häufigsten Nebenwirkungen vom Grad 3/4 waren Neutropenie (83\% unter der Kombination vs. $86 \%$ unter Topotecan-Monotherapie), Anämie (84 vs. $28 \%$ ) und Leukopenie (80 vs. 51 \%). Häufiger unter der Kombination waren zudem febrile Neutropenien (31 vs. $7 \%$ ) sowie Thrombozytopenien vom Grad 3/4 (41 vs. $28 \%$ ).

Fazit: Die Kombination aus Cisplatin, Etoposid, Irinotecan und G-CSF war bei Patienten mit sensitivem Rezidiv eines SCLC der Monotherapie mit Topotecan überlegen. Sie verlängerte das 6-MonatsÜberleben substanziell. Trotz der ernsten Nebenwirkungen unter der Kombinationschemotherapie könnte sie daher die neue Zweitlinienbehandlung für diese Patienten werden. Christina Berndt

Goto K et al. Combined chemotherapy with cisplatin, etoposide, and irinotecan versus topotecan alone as second-line treatment for patients with sensitive relapsed small-cell lung cancer (JCOG0605): a multicentre, open-label, randomised phase 3 trial. Lancet Oncol. 2016; 17(8):1147-57. 